University of Nebraska - Lincoln

DigitalCommons@University of Nebraska - Lincoln

Faculty Publications: Materials Research

Science and Engineering Center

Materials Research Science and Engineering

Center

2007

\title{
Cluster-Assembled Iron-Platinum Nanocomposite Permanent Magnets
}

\author{
Xiangxin Rui \\ University of Nebraska - Lincoln \\ Zhiguang Sun \\ University of Nebraska - Lincoln, zsun3@unl.edu \\ Yinfan Xu \\ University of Nebraska - Lincoln, yxu2@unl.edu \\ David J. Sellmyer \\ University of Nebraska-Lincoln, dsellmyer@unl.edu \\ Jeffrey E. Shield \\ University of Nebraska - Lincoln, jshield@unl.edu
}

Follow this and additional works at: https://digitalcommons.unl.edu/mrsecfacpubs

Part of the Materials Science and Engineering Commons

Rui, Xiangxin; Sun, Zhiguang; Xu, Yinfan; Sellmyer, David J.; and Shield, Jeffrey E., "Cluster-Assembled IronPlatinum Nanocomposite Permanent Magnets" (2007). Faculty Publications: Materials Research Science and Engineering Center. 80.

https://digitalcommons.unl.edu/mrsecfacpubs/80

This Article is brought to you for free and open access by the Materials Research Science and Engineering Center at DigitalCommons@University of Nebraska - Lincoln. It has been accepted for inclusion in Faculty Publications: Materials Research Science and Engineering Center by an authorized administrator of DigitalCommons@University of Nebraska - Lincoln. 


\title{
Cluster-Assembled Iron-Platinum Nanocomposite Permanent Magnets
}

Xiangxin Rui ${ }^{1}$, Zhiguang Sun $^{2}$, Yingfan Xu ${ }^{2}$, David J. Sellmyer ${ }^{2}$, and Jeffrey E. Shield ${ }^{1}$

${ }^{1}$ Mechanical Engineering, University of Nebraska-Lincoln, Lincoln, NE, 68588

${ }^{2}$ Physics and Astronomy, University of Nebraska-Lincoln, Lincoln, NE, 68588

\begin{abstract}
Exchange-spring nanocomposite permanent magnets have received a great deal of attention for their potential for improved the energy products. Predicted results, however, has been elusive. Optimal properties rely on a uniformly fine nanostructure. Particularly, the soft magnetic phase must be below approximately $10 \mathrm{~nm}$ to ensure complete exchange coupling. Inert gas condensation (IGC) is an ideal processing route to produce sub-10 $\mathrm{nm}$ clusters method. Two distinct nanostructures have been produced. In the first, Fe clusters were embedded in an $\mathrm{FePt}$ matrix by alternate deposition from two sources. Fe cluster content ranged from 0 to 30 volume percent. Post-deposition multi-step heat treatments converted the FePt from the $\mathrm{A} 1$ to $\mathrm{Ll}_{0}$ structure. An energy product of approximately 21 MGOe was achieved. Properties deteriorated rapidly at cluster concentrations above 14 volume due to uncoupled soft magnetic regions (from cluster-cluster contacts) and cooperative reversal. The second nanostructure, designed to overcome those disadvantages, involved intra-cluster structuring. Here, Fe-rich Fe-Pt clusters separated by $\mathrm{C}$ or $\mathrm{SiO}_{2}$ were fabricated. Phase separation into $\mathrm{Fe}_{3} \mathrm{Pt}$ and $\mathrm{FePt}$ and ordering was induced during post-deposition multi-step heat treatments. By confining the soft and hard phases to individual clusters, full exchange coupling was accomplished and cooperative reversal between clusters was effectively eliminated. An energy product of more than $25 \mathrm{MGOe}$ was achieved, and the volume fraction of the soft phase was increased to greater than 0.5 while maintaining a coercivity of $6.5 \mathrm{kOe}$. The results provide new insight into developing high energy product nanostructured permanent magnets.
\end{abstract}

\section{INTRODUCTION}

The exchange-spring phenomenon has received significant attention because of its potential to increase the energy densities of permanent magnets [1,2]. Combining soft and hard magnetic phases together at the nanoscale results in a very high remanent magnetization while maintaining sufficient coercivity, producing a high energy product. To ensure effective exchange coupling, the dimension of the soft magnetic phase should be on the order of twice the domain wall width of the hard magnetic phase, which is usually less than $10 \mathrm{~nm}$.

Practically, it is difficult to obtain an ideal nanostructure. For conventional processing methods like melt-spinning [3,4] or mechanical milling[5], the sizes of grains are larger than $15 \mathrm{~nm}$. This results in incomplete exchange coupling, leading to early magnetic reversal and dramatic losses of coercivity. Materials processing utilizing gas aggregation offers the opportunity to produce a uniformly fine-scale structure below $10 \mathrm{~nm}$ [6]. Here, we combine gas aggregation with conventional thin film deposition techniques to produce soft phase regions below $10 \mathrm{~nm}$, 
ensuring excellent exchange coupling and resulting in energy products above 20 MGOe for isotropic systems.

\section{EXPERIMENTAL PROCEDURES}

\section{Experimental setup}

For the gas-aggregation technique, the base pressure of the system (shown in Fig. 1.) was below $10^{-7}$ Torr. Argon/Helium (Ar/He) gas mixture was introduced into the system during cluster preparation. The atomic gas was produced by a 3 inch DC magnetron sputtering gun first, and then clusters were formed through collisions with Ar ions at a temperature of approximately $-130^{\circ} \mathrm{C}$. He gas was used to ensure uniform temperature in the nucleation chamber. For some experiments, a mass selector was used to better control the cluster size.

The gas aggregation system is also equipped with a second DC magnetron sputtering source, which can be used to deposit metal films such as Ag [7], FePt,

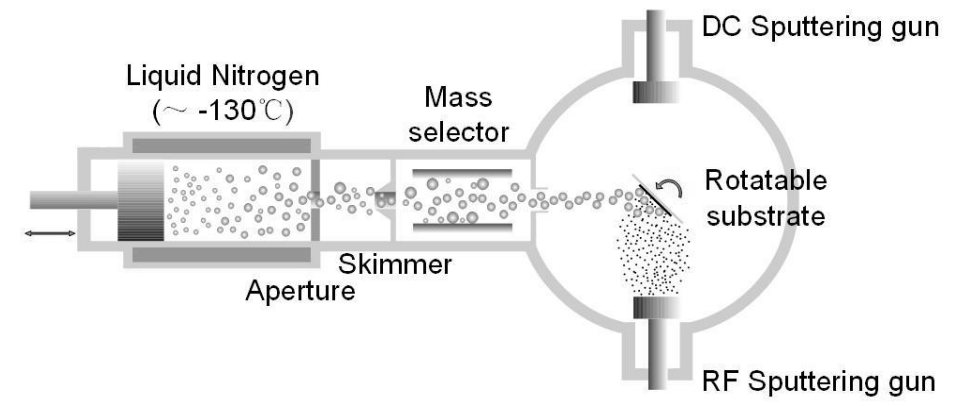

Fig. 1. A schematic picture of gas-aggregation system or $\mathrm{Cu}$, and an $\mathrm{RF}$ magnetron sputtering source, which can deposit nonmetallic thin films such as amorphous carbon [7] or $\mathrm{SiO}_{2}$.

\section{Fe-FePt system}

For this system, the Fe cluster was made from a $99.95 \%$ pure Fe target. By varying the Ar and He gas flow rates as well as the sputtering power, the cluster size and size distribution were well controlled. An FePt thin film was made from a composite target being sputtered from the second DC gun. By alternating deposition between the cluster gun and the second DC gun, the nanocomposite structures were achieved. Different phase contents in the nanocomposite structures were achieved by varying the deposition times for the two sources. The as-deposited FePt film was in the disordered A1 structure; multi-stage heat treatments were used to form the hard magnetic $\mathrm{L}_{0}$ structure after the deposition using Rapid Thermal Annealing (RTA) in flowing Ar with a ramp rate of $200^{\circ} \mathrm{C} / \mathrm{s}$.

\section{FePt $-\mathrm{Fe}_{3}$ Pt system}

To confine soft $\mathrm{Fe}_{3} \mathrm{Pt}$ and hard FePt within one cluster, the Fe-rich Fe-Pt cluster was made from a composite target consisting of $99.99 \%$ pure Pt pellets in a $99.95 \%$ pure Fe target. The composition of the clusters was controlled by varying the number of Pt pellets in the target. By physically rotating the substrate for deposition from the clusters and $\mathrm{C}$ or $\mathrm{SiO}_{2}$ thin film source, the nanocomposite structures were achieved. A mass selector was used during the cluster preparation to better control the size and size distribution of clusters. The as-deposited clusters were in the disordered A1 structure. Multi-stage heat treatments again using RTA were applied to accomplish the phase separation and disorder-order transformation for $\mathrm{Fe}_{3} \mathrm{Pt}$ and $\mathrm{FePt}$ respectively.

\section{Characterization}




\section{Characterization}

The clusters and nanocomposite structures were characterized by transmission electron microscopy (TEM) using a JEOL2010 TEM operating at $200 \mathrm{kV}$. X-ray diffraction data were collected using a Rigaku D/Max-B diffractometer with $\theta-\theta$ geometry. Magnetic properties were measured by a Quantum Design Magnetic Property Measurement System (MPMS) SQUID magnetometer and an alternating gradient field magnetometer. The magnetic signal from the diamagnetic Si substrate was subtracted from the magnetic signal by fitting a straight line to the high-field region and subtracting the linear portion from the measured signal. Deposition rates were measured in situ using a quartz crystal thickness monitor, and were also cross-checked ex situ using transmission electron microscopy by determining the number of clusters deposited per unit area per time. All results of magnetic properties are for in-plane measurements only, so the demagnetization factor was neglected.

\section{RESULTS AND DISCUSSIONS}

\section{Fe-FePt system}

Nearly monodispersed $8 \mathrm{~nm}$ Fe clusters were produced by gas aggregation by carefully selecting the processing parameters. The as-deposited Fe clusters were confirmed to be BCC $\alpha$ Fe by electron diffraction; Nanocomposite structures consisting of Fe clusters imbedded in an FePt matrix were fabricated by alternate deposition from two sources. The volume fraction of $\mathrm{Fe}$ clusters was adjusted from $0 \%$ to $30 \%$ by varying the deposition times for the Fe clusters while keeping the total FePt thickness constant. Numerous heat treatment times and temperatures were examined, but annealing at $600^{\circ} \mathrm{C}$ for 10 minutes resulted in the highest coercivity values (above $10 \mathrm{kOe}$ ). In all cases the structure displayed random crystallographic orientation.Structural characterization of the nanocomposite structure proved difficult because of the complex microstructure associated with the ordering process. An analysis of the diffusion profile after heat treatment [8] suggested that the heat treatments did not result in dissolution of the $\mathrm{Fe}$ clusters.

The magnetic properties depended strongly on the Fe cluster content. The coercivity of the nanocomposite structures decreased with increasing Fe cluster content, while the remanence increased systematically. The energy product increased, reaching a maximum of 17.7 MGOe at 8 volume percent Fe clusters, and then decreased with increasing Fe Cluster content (Fig. 2.).

The effect of multi-step heat

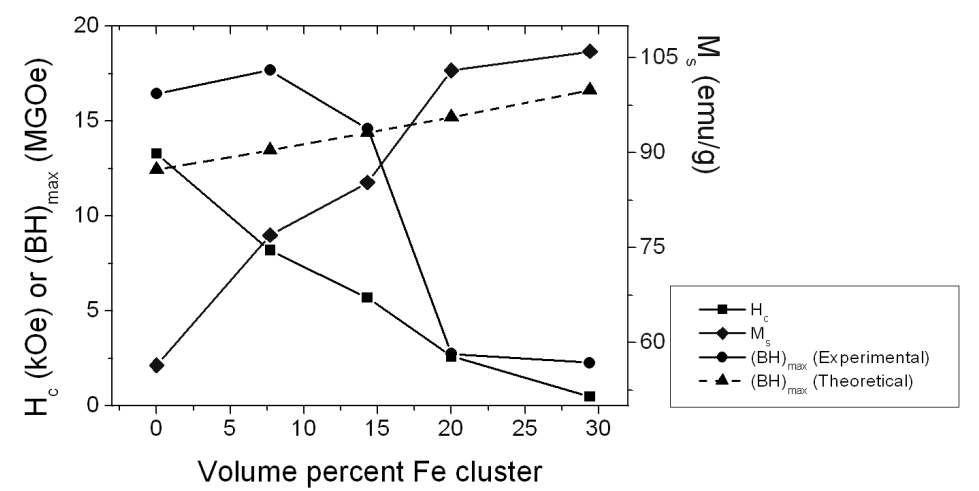

Fig. 2. Summary of magnetic properties of $\alpha$-Fe clusters/FePt nanocomposite structures treatment on the magnetic properties was also investigated. After heat treatment at $600^{\circ} \mathrm{C}$ for 10 minutes, samples with 8 and 14 volume percent Fe clusters were heat treated at $500^{\circ} \mathrm{C}$ for 10 and 20 minutes. The additional heat treatments effectively eliminated the step in the second quadrant of the hysteresis loop that was present after the initial heat treatment. This resulted in coercivity and remanence enhancement for both samples (Fig. 3.), resulting in an increase of the energy 
product to 20.7 MGOe. The enhancement in magnetic properties is likely due to the further ordering of the $\mathrm{L}_{0}$ structure, although a modification of the cluster/matrix interface structure may also contribute to the improved properties $[9,10]$.

The theoretical maximum energy product for a non-interacting, isotropic system can be calculated from the equation 1 .

$$
(B H)_{\max }=\left(4 \pi M_{s}\right)^{2} / 16
$$

. Calculating $4 \pi M_{s}$ using a rule of mixtures approach and saturation values of $14.1 \mathrm{kG}$ for the FePt $\mathrm{L} 1_{0}$ structure and 21.6 $\mathrm{kG}$ for $\alpha$-Fe results energy products of 13.84 MGOe and 14.67 MGOe for the 8 and 14 volume percent Fe cluster nanocomposites. The variation of energy product with cluster content for this model is shown by the dashed line in Figure 2. Clearly, the

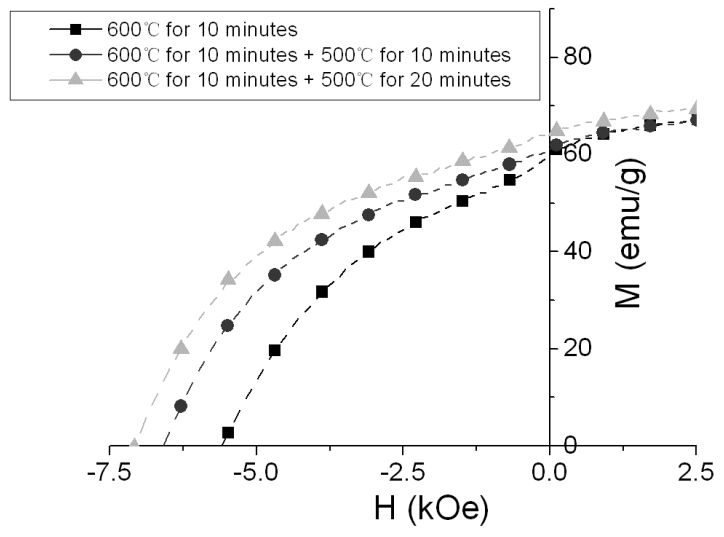

Fig. 3. Demagnetization curves of the 14 volume percent Fe clusters/FePt nanocomposite heat treated at various conditions nanocomposite structures have improved magnetic properties that arise from exchange interactions between the hard and soft magnetic phases.

Although relatively high energy products have been achieved by embedding $8 \mathrm{~nm}$ Fe clusters in an FePt matrix, the volume fraction of clusters is limited to about 14 percent. At Fe cluster contents above this, a dramatic decrease in coercivity was observed. This soft magnetic phase limit is similar to what has been observed in other granular exchange-spring magnets. It arises from the granular nature of the microstructure. Grains typically have 14 sides; thus, one soft grain with 14 sides can be completely surrounded by hard magnetic grains. Assuming equal grain sizes, this corresponds to 6.7 volume percent soft phase. At twice that volume fraction (13.4 percent), it is statistically probable that there will be contact between two soft magnetic grains. These "double grains" will have magnetically uncoupled regions that can easily reverse, resulting in dramatic decreases in coercivity. The reversed regions then expand due to cooperative reversal that arises from the strong intergranular interactions. To alleviate these problems, we have developed intra-cluster structured Fe-Pt consisting of $\mathrm{Fe}_{3} \mathrm{Pt}$ and $\mathrm{FePt}$ phases [11].

\section{Fe 3 Pt-FePt system}

Fe-Pt system is an excellent candidate for producing two-phase structures containing soft and hard phases. As shown in the phase diagram [12], soft $\mathrm{Fe}_{3} \mathrm{Pt}$ and hard FePt coexist between 29 and 37 atomic percent Pt. Samples of $6 \mathrm{~nm}$ Fe-rich Fe-Pt clusters were imbedded in $\mathrm{C}$ or $\mathrm{SiO} 2$ matrix by alternate deposition between the cluster gun and the RF gun (fig. 4). Although the deposition conditions led to some particleparticle contacts, clusters were largely

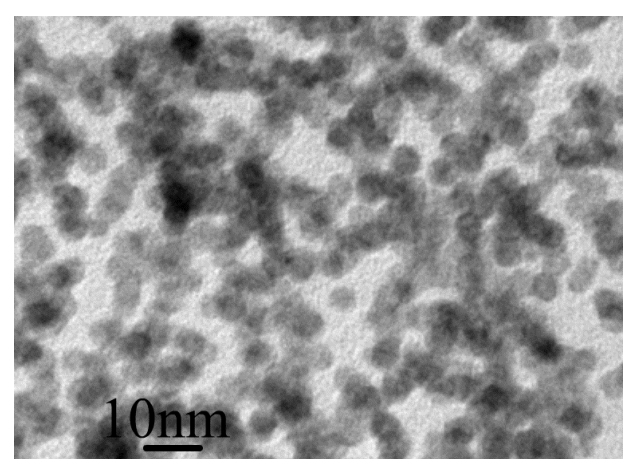

Fig. 4. As-deposited Fe rich Fe-Pt clusters isolated. The Pt content was adjusted from 26 to 42.2 atomic percent. 
The as-deposit samples that lie in the two-phase region formed in the A1 structure. Initial heat treatment at $580^{\circ} \mathrm{C}$ for 10 minutes did little to improve the coercivity, indicating that the $\mathrm{L} 1_{0}$

phase had not yet formed. Subsequent heat treatment at $550^{\circ} \mathrm{C}$ for 30 minutes and then $600^{\circ} \mathrm{C}$ for 20 minutes led to the development of significant coercivity $(>6$ $\mathrm{kOe})$ in some materials. Rietveld analysis of x-ray diffraction data of the fullyannealed sample with 33.1 atomic percent $\mathrm{Pt}$ revealed that ordered $\mathrm{FePt}$ and ordered $\mathrm{Fe}_{3} \mathrm{Pt}$ coexist in the sample (Figure 5). The two phases could be best identified in the twotheta region around the $\{002\}$ diffraction peaks. During the ordering of FePt from A1 structure to $\mathrm{L} 1_{0}$ structure, the original $\{002\}$ peak split into $\{200\}$ and (002) peaks (fig. 5

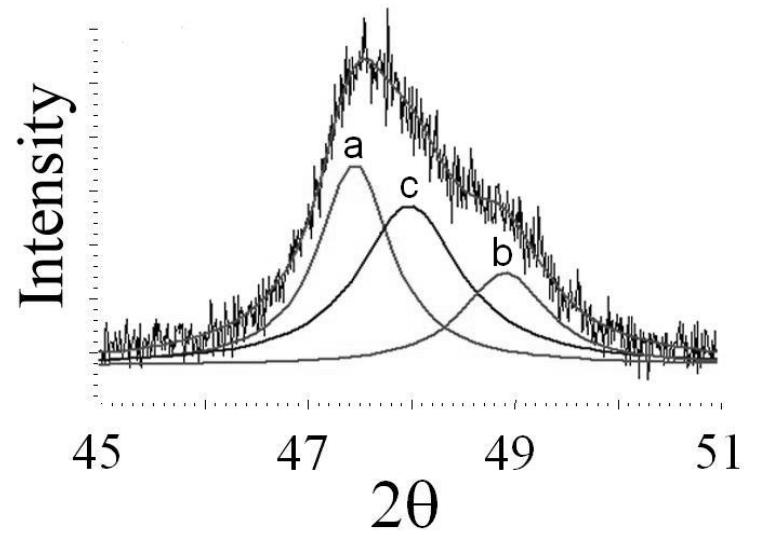

Fig. 5. Rietveld simulation of x-ray pattern of sample $\mathrm{Fe}_{67} \mathrm{Pt}_{33}$ (a), (b)), while the $\{002\}$ peak (fig 5. (c)) for $\mathrm{Fe}_{3} \mathrm{Pt}$ lies in between the $\{200\}$ and (002) peaks of FePt. The percentage of each of the phases can also be determined from the diffraction peak intensities, which for the 33.1 atomic percent Pt alloy was 55 percent soft magnetic $\mathrm{Fe}_{3} \mathrm{Pt}$ and 45 percent hard magnetic FePt, similar to Lever Rule calculations and significantly higher than previously achieved.

Magnetic properties measurements showed that, for the two-phase samples, the magnetization changed uniformly in the second quadrant of the hysteresis loop, acting like a single-phase material (Fig. 6 (a)). This indicates strong exchangecoupling between hard and soft phases within each cluster. This is in comparison to the Fe cluster-FePt nanocomposite, which displayed steps in the second quadrant (fig. 6 (b)) indicative of the presence of uncoupled soft magnetic regions.

For single-phase FePt and $\mathrm{Fe}_{3} \mathrm{Pt}$ clusters,

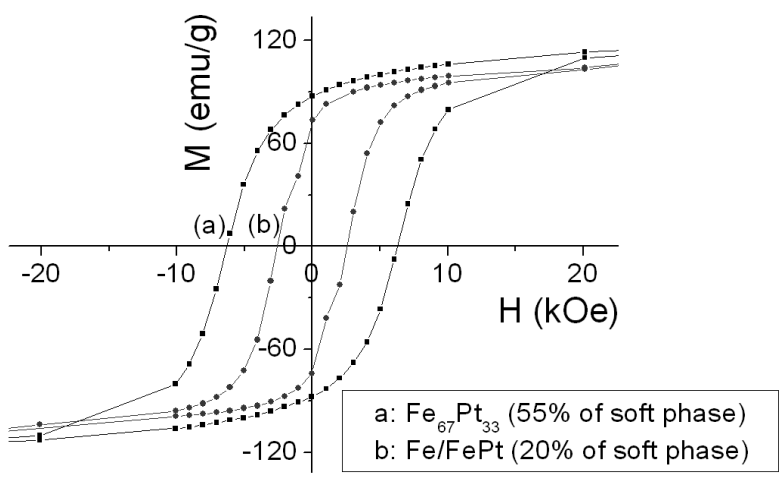

Fig. 6. Hysteresis loops of (a) $\mathrm{Fe}_{67} \mathrm{Pt}_{33}$ and (b) $\mathrm{Fe} / \mathrm{FePt}$ with $20 \%$ of Fe cluster the magnetic properties changed very little between the first and second heat treatments. This is because only ordering is required to form the $\mathrm{L} 1_{0}$ or $\mathrm{L}_{2}$ structures, which requires atomic reordering on a local scale. On the other hand, the magnetic properties of the clusters with compositions in the two-phase region remained low. However, a significant improvement in the magnetic properties, particularly the coercivity, was observed after further annealing at $600^{\circ} \mathrm{C}$ for 10 and 20 minutes. The additional heat treatments were necessary to form the two-phase structure from the parent, compositionally homogeneous A1 structure because phase separation requiring atomic diffusion over a (relatively) longer scale is necessary. The maximum coercivity was improved from $<3 \mathrm{kOe}$ to more than $6 \mathrm{kOe}$. Furthermore, the energy product improved to 25.1 MGOe, 25 percent higher than previously reported for the $\mathrm{Fe}_{3} \mathrm{Pt} / \mathrm{FePt}$ system [2] or $\mathrm{Fe} / \mathrm{FePt}$ system [8], and the highest ever reported for isotropic Fe-Pt alloys. 


\section{CONCLUSIONS}

Sub-ten nanometer Fe and Fe-Pt clusters were fabricated by the gas aggregation method. The size and size distribution of the clusters were controlled by adjusting the Ar/He gas flow rate and the sputtering power. Further size selection was achieved by employing a mass selector. Two structures of nanocomposite were made: one with Fe clusters embedded in an FePt matrix, and one through intracluster structuring with $\mathrm{Fe}_{3} \mathrm{Pt}$ and $\mathrm{FePt}$ phases present within one cluster, with the clusters initially imbedded in a non-magnetic matrix. By varying the composition ratio between Fe cluster and $\mathrm{FePt}$ matrix or between $\mathrm{Fe}_{3} \mathrm{Pt}$ and $\mathrm{FePt}$, a maximum energy product of 20.7 MGOe can be achieved for the first nanocomposite structure, and 25.1 MGOe for the second structure with intracluster structuring. Compared with the first structure, the second structure, with intracluster exchange-coupling, has the advantages that uncoupled regions and cooperative reversal could be avoided, resulting in a higher fraction of soft phase (more than 50\%). The extremely high soft magnetic phase content resulted in an increase of the remanent magnetization while maintaining a high coercivity, ultimately producing record-high energy products.

\section{ACKNOWLEDGMENTS}

The authors appreciate support from the national Science Foundation through MRSEC: QSPINS (Grant no. 0213808), the Army Research Office, Department of Energy, and the Nebraska Center for Materials and Nanoscience.

\section{REFERENCES}

1. R. Skomski, J. M. D. Coey, Phys. Rev. B, 48, 15812 (1993).

2. H. Zeng, J. Li, J. P. Liu, Z. L. Wang, and S. Sun, Nature, 420, 395 (2002).

3. J. E. Shield, J. Zhou, S. Aich, V. Ravindran, R. Skomski, and D. J. Sellmyer, J. Appl. Phys. 99, 08 B508 (2006).

4. W. Zhang, P. Sharma, K. Shin, et.al., Scripta Materialia, 54, 431 (2006).

5. J. Lyubina, I Opable, K.H.Muller, et.al., J. Phys. : Condens. Matter, 17, 4157 (2005).

6. H. Haberland, M. Karrais, M. Mall, and Y. Thurner, J. Vac. Sci. Technol. A10, 3266 (1992).

7. D. J. Sellmyer, M. Yan, Y. Xu, and R. Skomski, IEEE Trans. Magn., 41, 560 (2005).

8. X. Rui, Y. Xu, Z. Sun, D. J. Sellmyer, Z. Liu, D. J. Miller, and J. E. Shield, J. Magn. Magn. Mater. 305, 76 (2006).

9. D.C. Crew, L.H. Lewis, J. Kim, K. Barmak, J. Appl. Phys. 897528 (2001).

10. J.S. Jiang, J.E. Pearson, Z.Y. Liu, B. Kabius, S. Trasobares, D.J. Miller, S.D. Bader, D.R. Lee, D. Haskel, G. Srajer, J.P. Liu, J. Appl. Phys., 97 10K311 (2005).

11. X. Rui, J.E.Shield, Z.Sun, Y.Xu, D.J.Sellmyer. App. Phys.Lett. 89, 122509 (2006).

12. W. G. Moffatt, "The Handbook of Binary Phase Diagrams", Genium Publishing Corporation, 3, 7/84 (1984) 